\title{
Diversité génétique et dynamique des formes adventices de riz de camargue
}

\author{
Cécile Bres-Patry, Martine Bangratz, Alain Ghesquière*
}

Laboratoire Genetrop, Institut de recherches pour le développement, BP 5045, 34032 Montpellier Cedex, France

\begin{abstract}
Résumé - À côté des riz adventices des régions tropicales qui résultent d'introgressions variées avec les riz sauvages, de nouvelles formes ont émergé plus récemment et présentent aussi de nombreux attributs des formes typiquement spontanées d'O. rufipogon (égrenage, dormance, aristation, pigmentation des organes). Cette apparition concerne de nombreuses régions du monde pratiquant la riziculture intensive avec semis direct et situées le plus souvent en dehors de la zone de répartition géographique d'O. rufipogon. Différents échantillons d'adventices provenant de Camargue ont été analysés pour 15 marqueurs microsatellites comparativement à une collection représentative de 145 variétés de riz cultivé d'origine asiatique. La diversité génétique des riz adventices est cohérente avec la structuration d' $O$. sativa qui est organisée en deux groupes principaux indica et japonica. Les adventices de Camargue montrent une diversité plus importante que les variétés locales (japonica tempérées) ainsi que la présence d'allèles originaux ou plus caractéristiques des variétés indica. Cette diversité est totalement indépendante des variétés cultivées localement. La structure génétique de ces formes ne semble pas liée à des flux de gènes avec les variétés dont elles sont isolées grâce à une précocité plus forte mais à une dynamique autonome de ces formes due à des possibilités de conservation (dormance) et de dissémination des graines (égrenage). Le rôle des croisements éloignés $($ indica $\times$ japonica $)$ sur la structure et l'expression du génome pourrait être une explication possible à ce processus.
\end{abstract}

riz adventices / Oryza sativa L. / microsatellites / diversité génétique

Abstract - Genetic diversity and population dynamics of weedy rice in
Camargue area. Weedy rice have long been known, particularly in tropical areas,
as the result of introgressions between wild and cultivated forms. More recently, rice
fields have been frequently invaded by new weedy forms in temperate regions where
wild forms were never reported. These plants show many traits similar to true wild
O. rufipogon (shattering, dormancy, awning, organ pigmentation). Their occurrence

* Correspondance et tirés-à-part

E-mail: alain.ghesquiere@mpl.ird.fr 
concerns many parts of the world practicing intensive rice-growing with direct seeding. Samples of weeds from Camargue have been analyzed with 15 microsatellite markers in comparison to a representative collection of 145 Asian rice varieties. Diversity of weedy rice is in agreement with the genetic structure of $O$. sativa mainly organized in two main groups of varieties, indica and japonica. Weeds from Camargue showed a greater diversity than Mediterranean varieties (temperate japonica) as well as the presence of original alleles or alleles more typical of indica varieties. Genetic structure of weed stands appeared more probably the consequence of a predominantly autonomous dynamics allowed by seed conservation and dissemination than the result of gene flow with varieties from which they are isolated by a much greater precocity. These results suggested that distant crosses between indica and japonica varieties and their effect on genome structure and expression could be a relevant explanation to this "weediness" process.

sweedy rice / Oryza sativa L. / microsatellites / genetic diversity

\section{INTRODUCTION}

Les formes adventices de riz sont connues depuis très longtemps, notamment dans les régions tropicales telles que l'Asie du sud où elles résultent d'introgressions entre l'espèce sauvage $O$. rufipogon et l'espèce cultivée $O$. sativa. En Afrique, il existe également des formes adventices intermédiaires entre l'espèce africaine de riz cultivé, $O$. glaberrima et son ancêtre sauvage $O$. breviligulata, mais également entre les deux espèces cultivées $O$. sativa et $O$. glaberrima. Ces formes jouent un rôle primordial dans la domestication et l'évolution du pool cultivé (pour une revue : [5]). Ces dernières années, des riz adventices présentant de nombreux caractères analogues des formes sauvages d'O. rufipogon (égrenage, dormance, aristation, pigmentation des organes) ont envahi les rizières des régions tempérées. L'origine de ces formes adventices est d'autant plus mystérieuse qu'O. rufipogon est absente de ces régions et aucune étude n'a pu éclaircir jusqu'à présent les différents facteurs responsables de leur apparition. Les riz adventices peuvent, s'ils ne sont pas pris en compte et éliminés précocement, diminuer fortement les rendements des champs infestés. Ils constituent à l'heure actuelle l'un des problèmes majeurs de la riziculture irriguée intensive d'autant plus qu'il n'existe pratiquement aucun moyen de lutte efficace. Seules certaines pratiques culturales et l'élimination systématique de toute forme adventice avant leur floraison, permettent de réduire l'ampleur de ce phénomène.

Peu de travaux sur les formes adventices ont été menés jusqu'à présent $[4,11,12]$. Nous avons analysé dans cette étude plusieurs échantillons de riz adventice de Camargue à l'aide de marqueurs moléculaires très variables, les microsatellites. Le choix de ces marqueurs était justifié par la proximité génétique des variétés cultivées en Camargue qui appartiennent au groupe écomorphologique tempéré de la sous-espèce japonica. Cette analyse s'appuie sur l'étude d'une collection de 145 variétés de riz cultivé asiatique pour laquelle une 
grande partie des allèles microsatellites a été caractérisée [3]. Notre objectif est d'étudier la diversité génétique des formes adventices de Camargue et d'évaluer les hypothèses possibles pour expliquer leur origine à partir de l'observation de la dynamique de ces formes dans quelques situations représentatives de champs semenciers et de champs de production.

\section{MATÉRIELS ET MÉTHODES}

\subsection{Matériel végétal}

Les champs semenciers de Camargue sont des parcelles extrêmement contrôlées pour la production de semences de qualité (《terres neuves » non cultivées en riz depuis au moins 5 ans), utilisation de semences de base, épuration manuelle systématique des plantes adventices tout au long de la culture. Un champ semencier de la variété Thaïbonnet (T1) remplissant toutes ces caractéristiques a été échantillonné dans deux parcelles contiguës (T1a et T1b) qui ne présentaient que de très rares plantes adventices sous formes de taches isolées et dans une troisième parcelle (T1c) qui montrait plus d'adventices concentrées en bordure de champ. Les champs de production peuvent présenter des niveaux d'infestation très variables en fonction de l'efficacité des fauxsemis et des désherbages pratiqués par l'agriculteur. Les échantillons T2 et A1 ont été collectés dans deux champs de production voisins appartenant à la même exploitation agricole et respectivement cultivés avec les variétés Thaïbonnet et Ariete. Le champ A1 était extrêmement infesté par les riz adventices alors que le champ T2 avait été partiellement épuré. Un troisième échantillon d'adventices collectées dans la variété Ariete (A2) provenait d'une autre exploitation et manifestait le même taux d'infestation que le champ A1. Les trois sites $(\mathrm{T} 1-\mathrm{T} 2 / \mathrm{A} 1-\mathrm{A} 2)$ correspondaient à trois exploitations agricoles distantes de plusieurs kilomètres. Les critères de récolte de ces plantes sont basés sur la hauteur des plantes (supérieure à la variété cultivée), leur maturité plus précoce que pour la variété cultivée et l'égrenage. Toutes les plantes adventices des champs T1 et T2 ont été prélevées, alors que seul un sous-ensemble l'a été dans les champs A1 et A2. Un récapitulatif des différents champs est donné dans le tableau I et leur situation est présentée sur la figure 1. En outre, l'étude de deux échantillonnages provenant d'une région du Brésil où ne sont cultivées que des variétés indica (à la différence de la Camargue où ne sont cultivées que des variétés japonica) nous a permis de compléter cette étude. Sept plantes issues d'un champ cultivé avec la variété Vermelho et sept plantes provenant d'un champ cultivé avec la variété Preto ont été analysées. Ces deux variétés sont cultivées en monoculture sur de très grandes surfaces et depuis de nombreuses années dans la région où ont été récoltées les adventices. Les 11 principales variétés cultivées en Camargue ont été ajoutées à l'analyse pour compléter la collection de référence [3]. Enfin, le polymorphisme intravariétal 
Tableau I. Descriptif des différents champs prospectés et des 169 individus récoltés en vue du typage moléculaire. T1, T2, A1 et A2 correspondent aux champs de Camargue. V et $\mathrm{P}$ correspondent aux champs du Brésil.

\begin{tabular}{cccccc}
\hline \multicolumn{5}{c}{ Nombre de Nombre de } & Caractéristiques \\
Nom & Type de champ & parcelles & plantes & Type de plantes & adventices \\
\hline T1 & Semencier & 3 & 56 & A, C : plantes isolées & A, B : peu marquées* \\
& & $(\mathrm{a}, \mathrm{b}, \mathrm{c})$ & & $\mathrm{B}:$ tâche & $\mathrm{C}:$ très marquées** \\
T2 & Consommation & 1 & 12 & Plantes isolées & Peu marquées* \\
A1 & Consommation & 1 & 51 & Plantes isolées & Peu marquées* \\
A2 & Consommation & 1 & 36 & Tâches & Peu marquées* \\
V & Consommation & 1 & 7 & Plantes isolées & Très marquées** \\
P & Consommation & 1 & 7 & Plantes isolées & Très marquées** \\
\hline
\end{tabular}

* caractéristiques adventices peu prononcées (sub-aristation et peu de pigmentations anthocyanées).

** fortes caractéristiques adventices (aristation très prononcée et nombreuses pigmentations anthocyanées).

a été déterminé sur les deux variétés de référence de cette étude, Thaïbonnet et Ariete, à partir de la collecte de 100 panicules de chacune des variétés dans les champ T2 et A1.

\subsection{Marqueurs moléculaires}

La recherche de polymorphisme sur des individus aussi proches génétiquement que des variétés japonica tempérées telles que les variétés cultivées en France nous a amené à choisir préférentiellement les microsatellites, marqueurs hypervariables et hautement polymorphes. Le choix des locus et les techniques utilisées sont décrites par Bres-Patry et al. [3]. Les mêmes microsatellites ont été utilisés pour la caractérisation de la collection de 145 variétés de riz [3] et la caractérisation des plantes adventices.

\subsection{Analyse des données}

Pour chaque locus, les PIC (Polymorphism Information Content [1]) ont été calculés.

L'analyse de la structuration génétique des peuplements adventices de Camargue a permis d'évaluer les paramètres intra et interpopulations (dans notre cas interchamps) et de tester les hypothèses relatives aux flux de gènes. L'analyse a été réalisée grâce à l'estimation des $F$ statistiques $[14,15]$ : Fis et Fst. Les estimateurs utilisés sont $f$ pour Fis et $\theta$ (pour Fst [13] et ont été calculés à l'aide des logiciels Fstat 2.8 [7] et Genepop 3.1c [9]. Ces logiciels permettent de tester l'hypothèse nulle d'association au hasard des gamètes ou 

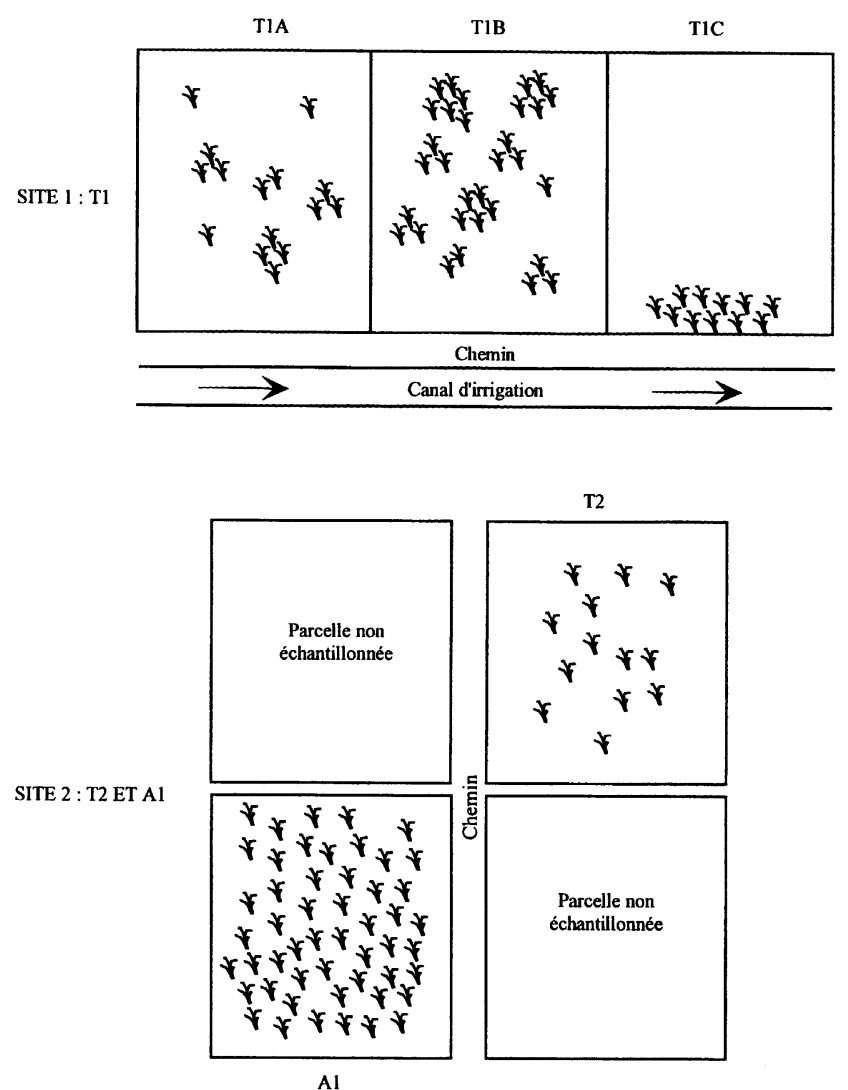

Al

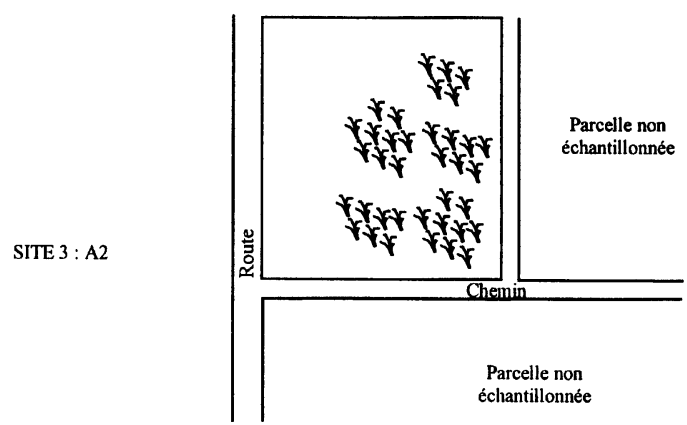

Figure 1. Localisation des différents sites de Camargue échantillonnés en 1997. Chaque symbole représente une plante adventice récoltée. Le site 1 correspond au champ T1, comprenant trois parcelles, a, b et c. Le site 2 correspond aux champs A1 et T2. Le site 3 correspond au champ A2. Les trois sites sont distants entre eux de quelques kilomètres. 
des génotypes, c'est-à-dire $f=0$ et $\theta=0$. La probabilité que les estimateurs soient supérieurs à zéro a été déterminée après 10000 permutations et un intervalle de confiance (95\%) a été déterminé après 15000 bootstraps [8]. Le taux d'allogamie a été calculé par la formule suivante : $(1-F i s) /(1+$ Fis $)$. Pour chaque paire de locus polymorphes, la signification statistique du déséquilibre de liaison a été testée dans la population globale et dans chacune des populations de Camargue. Une correction de Bonferroni a été appliquée pour la significativité des tests au niveau locus [8].

Des analyses en coordonnées principales sur matrices de distance ont été réalisées sur la base d'une distance génétique prenant en compte la taille de l'allèle microsatellite et correspondant au modèle de mutation « pas à pas » (Stepwise Mutation Model ou SMM [6]) qui a été montré comme le plus adéquat dans le cas du riz [3]. Cette distance correspond à la moyenne au carré de la différence du nombre de répétitions sur l'ensemble des locus.

\section{RÉSULTATS}

\section{1. Étude du polymorphisme microsatellite des formes adventices}

Afin d'évaluer la pertinence des marqueurs microsatellites choisis pour décrire la diversité des variétés cultivées en Camargue et interpréter celle des formes adventices, une étude préliminaire a été réalisée pour mesurer le polymorphisme intravariétal de Thaïbonnet et Ariete, variétés majoritairement cultivées depuis quelques années dans cette région. Sur 100 panicules collectées au hasard dans des champs de production de ces deux variétés, les 15 locus microsatellites choisis pour cette étude n'ont révélé aucune différence et tous les individus ne présentaient qu'une seule bande. Avec cet effectif, le taux de polymorphisme détectable au seuil $5 \%$ est seulement de $3 \times 10^{-2}$ et il a été considéré que l'homogénéité de ces deux variétés était suffisamment élevée pour caractériser la diversité des variétés méditerranéennes à partir d'un petit nombre de plantes.

Un total de 75 allèles a été détecté avec 15 locus microsatellites sur 169 plantes adventices. Le nombre d'allèles est compris entre un (locus RM019) et dix (locus RM001) avec une moyenne de cinq (Tab. II). En moyenne, le nombre d'allèles chez les plantes adventices est supérieur aux variétés méditerranéennes. Seuls quatre locus présentent un nombre d'allèles inférieur chez les formes adventices ou égal dans les deux groupes. Les valeurs moyennes des PIC sont équivalentes entre les plantes adventices et les variétés méditerranéennes mais cette valeur peut être très différente selon les locus considérés. Les plantes adventices montrent une plus grande diversité en terme de nombre d'allèles et de PIC mais également pour certains locus, une plus grande variation du nombre de répétitions que les variétés cultivées en Camargue (données non présentées). 
Tableau II. Nombre d'allèles et PIC (Polymorphism Information Content) des 15 locus microsatellites des 169 formes adventices et des 11 variétés méditerranéennes.

\begin{tabular}{|c|c|c|c|c|}
\hline & Populations & adventices & Variétés & méditerranéennes \\
\hline Locus & $N^{\mathrm{a}}$ & PIC & $N^{\mathrm{a}}$ & PIC \\
\hline RM001 & 10 & 0,74 & 4 & 0,70 \\
\hline RM005 & 4 & 0,50 & 2 & 0,32 \\
\hline RM011 & 5 & 0,56 & 2 & 0,30 \\
\hline RM018 & 9 & 0,77 & 3 & 0,43 \\
\hline RM019 & 1 & 0,00 & 2 & 0,46 \\
\hline RM021 & 9 & 0,72 & 4 & 0,45 \\
\hline RM022 & 6 & 0,26 & 4 & 0,66 \\
\hline RM148 & 2 & 0,34 & 2 & 0,30 \\
\hline RM167 & 6 & 0,23 & 3 & 0,59 \\
\hline RM168 & 4 & 0,55 & 3 & 0,63 \\
\hline RM231 & 2 & 0,23 & 3 & 0,54 \\
\hline RM232 & 8 & 0,61 & 2 & 0,42 \\
\hline RM246 & 4 & 0,39 & 3 & 0,43 \\
\hline RM252 & 2 & 0,14 & 2 & 0,30 \\
\hline RM255 & 3 & 0,25 & 1 & 0,00 \\
\hline Moyenne & 5 & 0,42 & 2,7 & 0,43 \\
\hline
\end{tabular}

$N^{\mathrm{a}}$ : nombre d'allèles.

L'analyse du polymorphisme microsatellite d'une collection d'O. sativa a permis d'identifier un grand nombre d'allèles microsatellites chez des variétés cultivées appartenant aux deux groupes variétaux indica et japonica [3]. Pour évaluer l'origine des allèles observés chez les plantes adventices, nous avons effectué une comparaison entre les allèles microsatellites observés au sein de cette collection et ceux observés chez les plantes adventices. Le tableau III montre les fréquences alléliques observées dans le groupe indica, le groupe japonica, chez les variétés méditerranéennes, les plantes adventices de Camargue et du Brésil. Sur l'ensemble des locus, les quelques variétés méditerranéennes présentent des allèles caractéristiques du groupe des variétés japonica tempérées. Elles peuvent aussi présenter, en fréquence élevée, des allèles qui sont rares dans le groupe japonica (RM021). Pour la majorité des locus, des allèles distinguent les adventices de Camargue des adventices du Brésil avec également des allèles communs aux deux groupes. Si on compare avec les variétés, la présence d'allèles plus caractéristiques des variétés indica (RM018) et d'allèles nouveaux 
Tableau III. Comparaison des fréquences alléliques des allèles microsatellites (en nombre de répétitions) entre les variétés du groupe indica, les variétés du groupe japonica, les variétés méditerranéennes, les plantes adventices de Camargue et les plantes adventices du Brésil. Pour une question de lisibilité, seuls les locus montrant des allèles supplémentaires non présents chez les formes adventices et seuls les allèles présents chez les plantes adventices sont notés.

\begin{tabular}{|c|c|c|c|c|c|c|}
\hline Locus & Allèle & $\begin{array}{l}\text { Groupe } \\
\text { indica }\end{array}$ & $\begin{array}{l}\text { Groupe } \\
\text { japonica }\end{array}$ & $\begin{array}{c}\text { Variétés } \\
\text { méditerranéennes }\end{array}$ & $\begin{array}{c}\text { Adventices de } \\
\text { Camargue }\end{array}$ & $\begin{array}{c}\text { Adventices du } \\
\text { Brésil }\end{array}$ \\
\hline \multirow[t]{10}{*}{ RM001 } & 10 & 0,15 & 0,05 & & 0,04 & 0,43 \\
\hline & 13 & & & & & 0,42 \\
\hline & 14 & 0,08 & 0,14 & 0,45 & 0,16 & 0,08 \\
\hline & 15 & 0,08 & 0,25 & 0,18 & 0,14 & \\
\hline & 16 & & 0,32 & & 0,08 & \\
\hline & 17 & & 0,12 & 0,18 & & \\
\hline & 18 & & & & 0,18 & \\
\hline & 19 & & 0,05 & 0,18 & 0,37 & \\
\hline & 20 & & & & 0,03 & \\
\hline & 26 & 0,41 & & & & 0,07 \\
\hline \multirow[t]{5}{*}{ RM011 } & 6 & & 0,04 & & 0,01 & 1,00 \\
\hline & 7 & 0,13 & 0,25 & 0,82 & 0,24 & \\
\hline & 8 & & 0,13 & & 0,53 & \\
\hline & 9 & & & & 0,08 & \\
\hline & 10 & 0,03 & 0,23 & 0,18 & 0,14 & \\
\hline \multirow[t]{9}{*}{ RM018 } & 12 & & & & 0,04 & \\
\hline & 15 & & & & 0,09 & \\
\hline & 16 & 0,39 & & & 0,03 & 0,10 \\
\hline & 17 & & 0,54 & 0,73 & 0,41 & 0,10 \\
\hline & 18 & 0,05 & 0,18 & & & 0,20 \\
\hline & 19 & & 0,09 & & 0,31 & 0,60 \\
\hline & 20 & & 0,14 & 0,09 & 0,02 & \\
\hline & 21 & & 0,03 & 0,18 & 0,09 & \\
\hline & 22 & & & & 0,01 & \\
\hline \multirow[t]{10}{*}{ RM021 } & 3 & 0,15 & 0,05 & & 0,10 & \\
\hline & 4 & 0,10 & 0,05 & & 0,40 & \\
\hline & 5 & & 0,05 & & 0,16 & 1,00 \\
\hline & 6 & & 0,08 & 0,09 & 0,07 & \\
\hline & 7 & & 0,14 & & 0,01 & \\
\hline & 8 & & 0,02 & 0,73 & 0,18 & \\
\hline & 9 & & & & 0,01 & \\
\hline & 14 & 0,03 & 0,20 & & 0,04 & \\
\hline & 16 & & 0,11 & 0,09 & & \\
\hline & 17 & & 0,08 & 0,09 & 0,04 & \\
\hline \multirow[t]{4}{*}{ RM246 } & 10 & & & & & 0,50 \\
\hline & 13 & & 0,43 & 0,18 & 0,66 & 0,37 \\
\hline & 15 & 0,50 & 0,34 & 0,09 & 0,11 & 0,13 \\
\hline & 16 & & & 0,73 & 0,23 & \\
\hline
\end{tabular}


Tableau IV. Nombre d'allèles moyens, diversité génétique $(H e)$, valeurs des $F i s(f)$ et probabilité des tests de significativité par population.

\begin{tabular}{cccc}
\hline Populations & $N^{\mathrm{a}}$ & $H e$ & $f(p=0,00)$ \\
\hline T1a & 2,26 & 0,31 & 1 \\
T1b & 1,46 & 0,12 & 1 \\
T1c & 2,4 & 0,41 & 1 \\
T2 & 2,4 & 0,43 & 1 \\
A1 & 3,25 & 0,36 & 1 \\
A2 & 2,63 & 0,44 & 0,92 \\
\hline
\end{tabular}

$N^{\mathrm{a}}$ : nombre d'allèles.

(RM001, RM011, RM018 et RM246) en fréquence faible, confirment la variabilité originale des adventices de Camargue par rapport aux variétés cultivées à proximité.

\subsection{Analyse de la structure de la variabilité génétique des adventices de Camargue}

\subsection{1. Étude de la structure de la variabilité génétique intrapopulation}

Comme cela est attendu dans des populations autogames, les estimations des Fis sont très hautement significatives et égales à 1 sauf pour une population, A2, qui montre une valeur inférieure à 1 tout en restant très hautement significative $(f=0,86$ et $f=0,43$ respectivement pour les locus RM001 et RM022 ; Tab. IV). Ces valeurs révèlent le mode de reproduction autogame mais quelques flux de gènes ont été mis en évidence. Le taux d'allogamie a été calculé pour cette population. Il est égal à $4,3 \%$ ce qui fait en moyenne sur l'ensemble des six populations adventices étudiées, un taux de 0,7\%. En outre, les déséquilibres de liaison, résultat de la structure de la variabilité entre populations (fréquences alléliques différentes entre populations) et de la structure des populations en lignées autogames, et calculés pour chaque paire de locus polymorphes et dans chacune des populations, sont très fréquents dans l'ensemble des populations (données non présentées).

\subsection{2. Étude de la structure de la variabilité génétique interpopulation}

Les valeurs de Fst ont été calculées par paires de champs sur l'ensemble des locus et sont très hautement significatives (Tab. V), ce qui montre une forte différenciation entre champs. Un arbre UPGMA a été construit à partir de la matrice des valeurs de $F s t$ (Fig. 4). Les champs proches en distance (T2 et A1) 
Tableau V. Matrice des estimateurs du Fst $(\theta)$ par paires de champs (probabilité des tests de significativité $p=0,00$ ).

\begin{tabular}{cccccc}
\hline & T1a & T1b & T1c & T2 & A1 \\
\hline T1b & 0,11 & & & & \\
T1c & 0,11 & 0,33 & & & \\
T2 & 0,38 & 0,63 & 0,28 & & \\
A1 & 0,14 & 0,32 & 0,15 & 0,41 & \\
A2 & 0,22 & 0,44 & 0,14 & 0,30 & 0,24 \\
\hline
\end{tabular}

ne se regroupent pas au sein de l'arbre. De même, les adventices provenant de champs cultivés avec la même variété (T1 et T2 ou A1 et A2) ne présentent pas de structuration particulière et ne montrent donc pas plus d'affinité entre elles que celles provenant de champs cultivés avec des variétés différentes. La proximité génétique des adventices collectées dans les champs A2 et T1c, bien qu'ils soient éloignés géographiquement et cultivés avec deux variétés différentes, semble plus liée à l'importance et à l'ancienneté de l'infestation.

\subsection{Analyses multivariées}

Les résultats des analyses multivariées sont présentés sur les figures 2 et 3 . Sur la figure 2, un échantillonnage de variétés représentatives des groupes indica et japonica a été utilisé pour positionner le matériel de Camargue. Les deux premiers axes représentent respectivement $42,1 \%$ et $21,6 \%$ de la variation totale. Les plantes adventices de Camargue se situent globalement dans le groupe des variétés japonica, mais certains génotypes ont une position plus intermédiaire et orientée vers le groupe indica. Les adventices du Brésil ont également une position intermédiaire entre les variétés indica et les variétés japonica. Sur la figure 3, où n'ont été analysées que les adventices et les variétés cultivées à proximité, cette tendance est confirmée. Les deux premiers axes représentent respectivement $41,5 \%$ et $27,8 \%$ de la variation totale. On observe un groupe de variétés méditerranéennes très proches avec une position plus éloignée des variétés Thaïbonnet et Inca. Les variétés du Brésil, Preto et Vermelho, contribuent très fortement à l'inertie de l'axe 1 et constituent une référence indica. Un premier groupe de plantes adventices (T1 et T2), récoltées dans des parcelles cultivées avec Thaïbonnet, présente une position très éloignée de cette variété et montrent au contraire un rapprochement avec d'autres variétés locales. Il semble qu'il n'y a pas de relation génétique entre les adventices et la variété cultivée à proximité. Un deuxième groupe, correspondant aux plantes adventices collectées dans les champs de production d'Ariete (A1 et A2), présente une diversité encore plus importante avec une position intermédiaire entre les plantes adventices des champs semenciers (T1 et T2) et les plantes adventices indica provenant du Brésil. Les génotypes du champ A2 


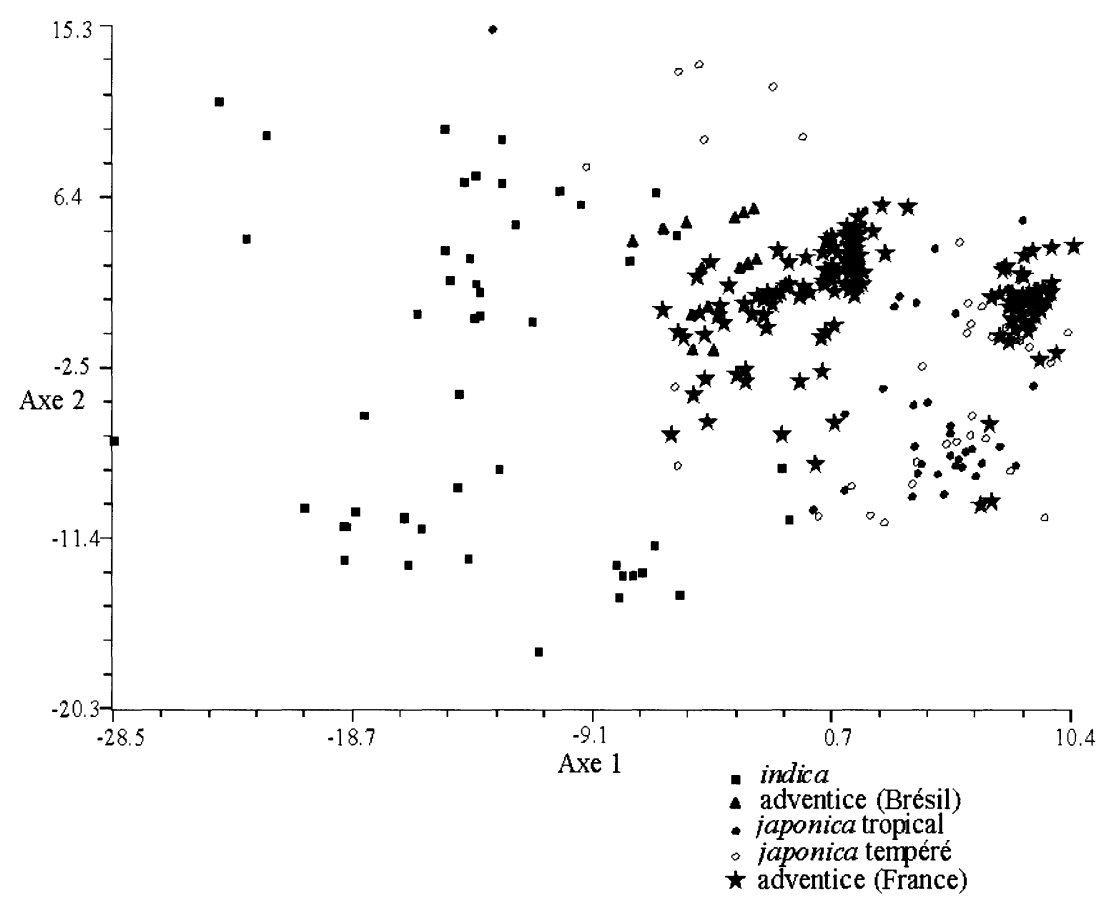

Figure 2. Analyse en coordonnées principales sur la matrice de distances basée sur le nombre de répétitions de 15 locus microsatellites. L'analyse porte sur des variétés indica et japonica de la collection d'O. sativa [3], les formes adventices de Camargue et les formes adventices du Brésil. Les axes 1 et 2 représentent respectivement $42,1 \%$ et $21,6 \%$ de la variation totale.

apparaissent comme les plus distants génétiquement. Ce champ présente le PIC le plus élevé mais également cinq individus hétérozygotes pour un ou plusieurs locus. Globalement, les adventices collectées dans les champs de Thaibonnet et d'Ariete apparaissent à la fois très différentes entre elles et très différentes de ces deux variétés.

\section{DISCUSSION}

Les résultats de l'analyse du polymorphisme microsatellite montrent une richesse allélique et une diversité très importante par rapport à celle que l'on attendait sur des plantes aussi proches génétiquement. Le nombre d'allèles observés chez les plantes adventices est plus important que chez les variétés méditerranéennes. La comparaison des allèles observés dans la collection d'O. sativa et chez les plantes adventices montre que ces allèles supplémentaires peuvent être plus fréquemment rencontrés dans les variétés japonica ou indica mais aucun d'entre eux ne peut réellement diagnostiquer une origine 


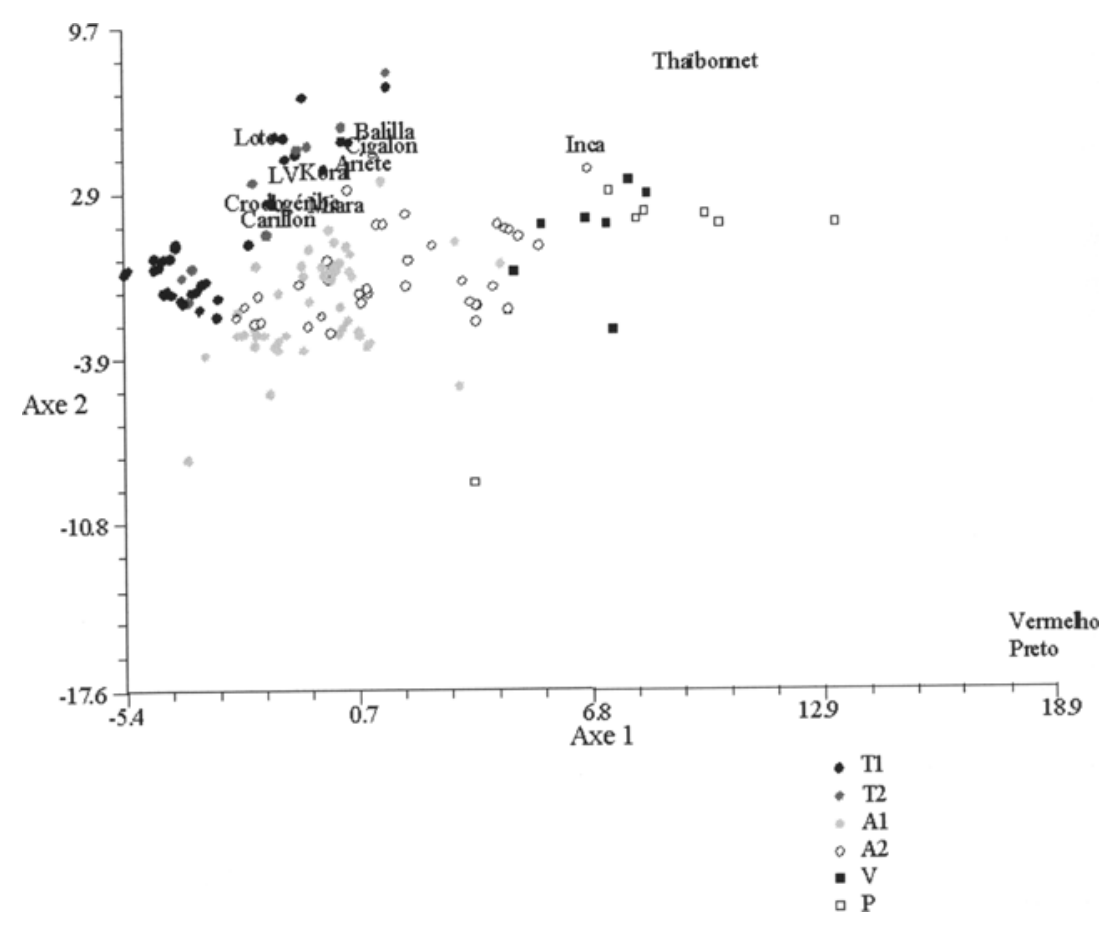

Figure 3. Analyse en coordonnées principales sur la matrice de distances basée sur le nombre de répétitions de 15 locus microsatellites. L'analyse porte sur les variétés méditerranéennes cultivées en Camargue, les variétés cultivées au Brésil et les formes adventices. Les axes 1 et 2 représentent respectivement $41,5 \%$ et $27,8 \%$ de la variation totale.

génétique spécifique. Également, des allèles « nouveaux», non répertoriés dans la collection et le plus souvent à très faible fréquence, ont été observés chez les adventices. Ces allèles originaux peuvent s'expliquer par la taille certainement insuffisante de la collection pour recenser de manière exhaustive l'ensemble des allèles microsatellites existant chez le riz cultivé d'origine asiatique. Dans la mesure où les variétés méditerranéennes constituent un ensemble génétique de plus faible diversité, il est possible d'associer des allèles microsatellites plus caractéristiques des variétés indica aux formes adventices de Camargue. Néanmoins, cette diversité se présente toujours sous forme fixée et n'a pas permis d'observer de génotypes multilocus hétérozygotes, caractéristiques d'hybridations récentes entre variétés indica et japonica. Les adventices de Camargue ont ainsi un fonds génétique de type japonica conforme à celui des variétés cultivées dans la région.

Dans l'état actuel des échantillonnages et alors que dans un champ semencier il semblait a priori plus favorable de mettre en évidence un lien entre variété 


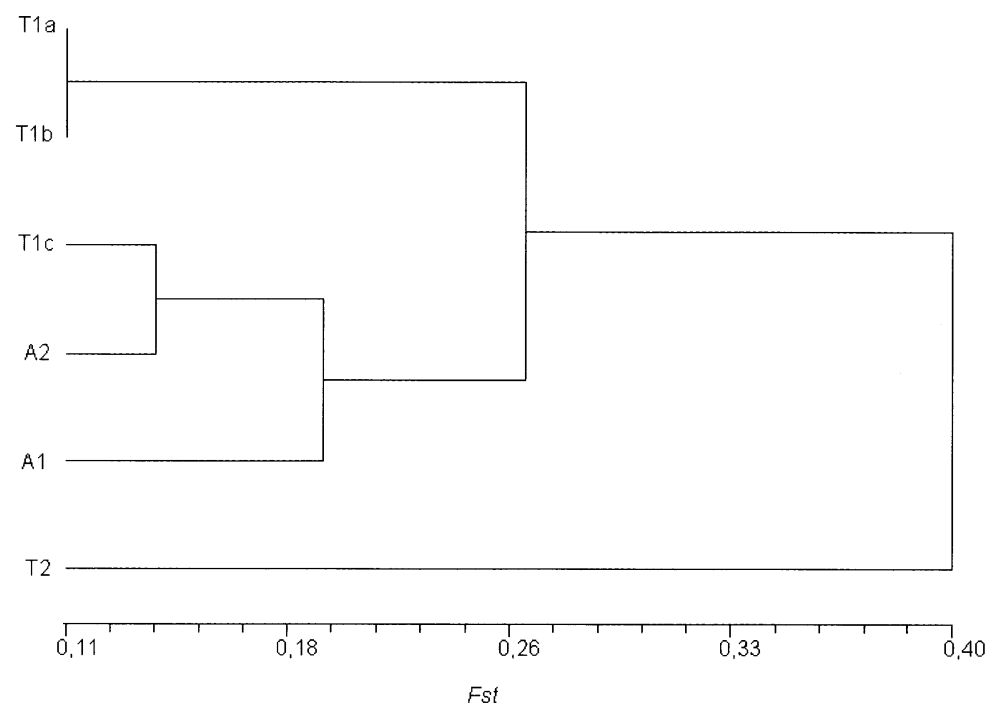

Figure 4. Arbre UPGMA basé sur la matrice de Fst entre champs.

cultivée et adventice, il ne nous pas été possible de mettre en relation la diversité génétique des formes adventices avec un facteur variétal ou un facteur géographique.

Les résultats de l'évaluation génétique des plantes adventices peuvent être confrontés aux données biologiques et aux observations de terrain pour essayer d'interpréter en terme de flux de gènes les deux types de situation analysés (champs semenciers/champs de production). Dans le champ semencier, les quelques génotypes analysés correspondent vraisemblablement à des plantes non épurées, probablement parce qu'elles manifestaient moins fortement ou plus tardivement ces caractéristiques. Les allèles différents vis-à-vis de la variété Thaïbonnet, montrent que ces plantes proviennent d'apports extérieurs. Par ailleurs, l'apparition plus diffuse des formes adventices dans des champs semenciers semble en faveur d'un apport direct par les semences. Cette observation peut être rapprochée du fait que les taux d'adventices détectés dans les champs semenciers sont très supérieurs au taux de graines adventices (à péricarpe rouge) toléré dans les lots de semence. Deux phénomènes, contamination externe par les pratiques culturales et présence de grains adventices dans les lots de semences peuvent donc expliquer cette diversité non apparentée aux variétés dans les toutes premières infestations. Dans les champs de production, la diversité est beaucoup plus importante que dans les champs semenciers, et peut témoigner de la mise en place d'une dynamique propre provenant de l'apport constant par les outils, de la récupération des phénomènes de dormance des graines qui permet leur stockage dans le sol et des possibilités de croisements. En effet, même si les plantes adventices montrent une très bonne fertilité et vraisemblablement une forte autogamie, des périodes de temps très 
sec coïncidant avec la floraison entraînent une stérilité et peuvent créer ainsi ponctuellement des conditions très favorables à l'allogamie.

Dans ces parcelles anciennes et fortement infestées, les adventices présentent une taille plus élevée et une précocité avancée d'une semaine à 15 jours. Cet aspect du décalage de la floraison entre adventices et variétés est sûrement important car, en déterminant les possibilités de croisement, il intervient sur la dynamique ultérieure des peuplements d'adventices. On admet qu'une précocité plus importante procure un avantage sélectif permettant d'assurer avant la récolte de la variété la dissémination des graines des plantes adventices et l'enrichissement de la banque de graines dans le sol. Ainsi, avec les infestations successives, la plus grande précocité des adventices va se traduire par un isolement reproductif efficace vis-à-vis des variétés cultivées et renforcé par l'autogamie des formes adventices. La sélection pour la précocité, l'égrenage et un fort potentiel de production de graines sont probablement les facteurs sélectifs principaux permettant d'aboutir à la convergence phénotypique caractéristique des champs fortement infestés. D'autre part, la cartographie génétique d'un croisement entre une variété méditerranéenne et une forme adventice a montré que les mêmes gènes/QTL sont impliqués dans le processus de domestication chez le riz et dans ce processus de dédomestication [2]. La colocalisation des caractères sur certains secteurs chromosomiques est un facteur favorable pour générer par recombinaison de nouvelles formes adventices à partir de croisements accidentels.

Ainsi, les apports par les semences et les apports accidentels par les outils sont probablement les deux facteurs principaux d'initiation du processus d'infestation. Bien que les marqueurs et les données biologiques ne sont pas en faveur d'un flux de gènes important des variétés vers les adventices, les rares croisements avec les variétés peuvent être suffisants pour accroître la diversité des adventices en générant des hybrides. L'évolution des premières infestations, si elle n'est pas maîtrisée, va dans le sens d'un isolement reproductif vis-à-vis des variétés au profit de croisements entre formes adventices et la possibilité d'enrichissement par de nouveaux apports extérieurs. La complexité de la diversité génétique des formes adventices est ainsi la résultante de ces phénomènes et suggère un fonctionnement en majeure partie autonome vis-à-vis des variétés cultivées. Il sera sans doute vain de rechercher une origine variétale particulière aux adventices collectées dans une variété donnée même si celle-ci est cultivée en monoculture depuis plusieurs années.

À cette dynamique propre des formes adventices qui dépend directement de leur valeur sélective et obéirait à certaines forces évolutives, on peut également s'interroger sur la structure et l'expression du génome de ces plantes. En l'absence de formes sauvages endémiques, il est tout à fait surprenant de voir apparaître de telles formes adventices même si on ne peut exclure définitivement l'introduction exceptionnelle de véritable formes adventices ou sauvages d'origine exotique. L'observation d'une diversité allélique plus caractéristique des variétés indica chez les formes adventices suggère que les croisements entre 
variétés indica et japonica pourraient expliquer l'origine première des formes adventices dans la mesure où expérimentalement, il est connu que les croisements éloignés chez le riz sont capables de restituer à des degrés divers des phénotypes de plantes adventices. On sait également que les sélectionneurs utilisent de plus en plus ces croisements pour accroître la variabilité de leur matériel et ils reconnaissent que certaines lignées sont particulièrement difficiles à fixer même lorsqu'elles sont issues d'androgénèse ou après plusieurs générations d'apparente homogénéité. Cette instabilité apparaissant à plusieurs générations de distance est d'autant plus cohérente avec la situation observée en Camargue que les croisements indica $\times$ japonica ne peuvent se faire en grande culture puisque les riziculteurs ne cultivent pas de variétés indica et que les formes adventices ont toujours un taux très élevé d'homozygotie. Enfin, il est possible aussi que des facteurs externes liés aux conditions limites de culture du riz (froid, par exemple) puissent également jouer un rôle de facteur déclenchant des modifications au niveau de la structure et/ou de l'expression du génome de certaines variétés et ceci bien après leur diffusion en grande culture.

\section{REMERCIEMENTS}

Cécile Bres-Patry a bénéficié d'une convention CIFRE entre les Semences de Provence, entreprise semencière d'Arles et le laboratoire Genetrop de l'IRD de Montpellier. Ce travail a été financé par le Bureau des ressources génétiques, le Centre français du riz et la Communauté européenne (FAIR CT96 1450 BICORER).

\section{RÉFÉRENCES}

[1] Botstein D., White R.L., Skolnick M., Davis R.W., Construction of a genetic linkage map in man using restriction fragment length polymorphism, Am. J. Hum. Genet. 32 (1980) 489-494.

[2] Bres-Patry C., Lorieux M., Clément G., Bangratz M., Ghesquière A., Heredity and mapping of domestication traits in temperate japonica weedy rice, Theor. Appl. Genet. 102 (2001) 118-126.

[3] Bres-Patry C., Second G., Rimbault I., Ghesquière A., Origin of the microsatellite polymorphism in cultivated Asian rice (Oryza sativa L.) through a comparison with RFLP marker diversity, Theor. Appl. Genet. (soumis).

[4] Cho Y.C., Chung T.Y., Suh H.S., Genetic characteristics of Korean weedy rice (Oryza sativa L.) by RFLP analysis, Euphytica 86 (1995) 103-110.

[5] Ellstrand N.C., Prentice H.C., Hancock J.F., gene flow and introgression from domesticated plants into their wild relatives, Annu. Rev. Ecol. Syst. 30 (1999) 539-563.

[6] Goldstein D.B., Linares A.R., Cavalli-Sforza L.L., Feldman M.W., An evaluation of genetic distances for use with microsatellite loci, Genetics 139 (1995) 463-471. 
[7] Goudet J., F-stat (version 1.2). A computer program to calculate F-statistics, J. Hered. 86 (1996) 485-486.

[8] Goudet J., Raymond M., de Meeüs T., Rousset F., Testing differentiation in diploid populations, Genetics 144 (1996) 1933-40.

[9] Raymond M., Rousset F., GENEPOP (version 1.2): population genetics software for exact tests and ecumenicism, J. Hered. 86 (1995) 248-249.

[10] Slatkin M., Isolation by the distance in equilibrium and non-equilibrium populations, Evolution 47 (1993) 264-279.

[11] Suh H.S., Sato Y.I., Morishima H., Genetic characterization of weedy rice (Oryza sativa L.) based on morpho-physiology, isozymes and RAPD markers, Theor. Appl. Genet. 94 (1997) 316-321.

[12] Tang L.H., Morishima H., Characteristics of weed rice strains, Rice Genet. News 5 (1988) 70-72.

[13] Weir B.S., Cockerham C.C., Estimating F-statistiques for the analysis of population structure, Evolution 38 (1984) 1358-1370.

[14] Wright S., The genetical structure of populations, Ann. Eugen. 15 (1951) 323354.

[15] Wright S., The interpretation of population structure by F-statistics with special regards to system of mating, Evolution 19 (1965) 395-420. 